DOI: https://doi.org/10.34069/AI/2021.46.10.8

How to Cite:

Kramar, N., \& Ilchenko, O. (2021). From intriguing to misleading: The ambivalent role of metaphor in modern astrophysical and cosmological terminology. Amazonia Investiga, 10(46), 92-100. https://doi.org/10.34069/AI/2021.46.10.8

\title{
From intriguing to misleading: The ambivalent role of metaphor in modern astrophysical and cosmological terminology
}

\section{Від інтригуючої до оманливої: амбівалентна роль метафори у сучасній термінології астрофізики та космології}

Received: September 15, 2021

\begin{abstract}
While metaphor has long been shown to pervade scientific discourse and terminology, little is known about how it affects the human comprehension of abstract concepts and underpins further development of related scientific ideas. In this article we focus on seven established terms in astrophysics and cosmology, which have also become staples of popular science, namely "big bang", "wormhole", "black hole", "spaghettification", "gravitational hair", "fuzzball", and "string" (in the context of string theory). We carry out etymological and contextual analysis to find out the specifics of their use in specialized and popular literature, with a particular emphasis on personification of physical entities. Also, we apply the conceptual metaphor theory to compare their source domain and target domains, identifying potentially misleading discrepancies between the two. We reveal that most of these metaphorical nominalizations invoke inaccurate and largely distorted images of the referenced entities, which are further extended and amplified with new details in scientific popularizations. We suggest that more research is needed into metaphorical terms in different disciplines to better understand their implications for the development of both expert and lay knowledge of the subject.
\end{abstract}

Accepted: October 28, 2021

\author{
Written by: \\ Natalie Kramar ${ }^{26}$ \\ https://orcid.org/0000-0001-5767-9200 \\ Olga Ilchenko ${ }^{27}$ \\ https://orcid.org/0000-0002-5546-1408
}

\footnotetext{
${ }^{26} \mathrm{PhD}$, Lecturer at the Research and Educational Center for Foreign Languages, National Academy of Sciences of Ukraine, Ukraine. ${ }^{27}$ Doctor of Philological Sciences, Professor, Head of the Department of Foreign Languages of the Research and Educational Center for Foreign Languages, National Academy of Sciences of Ukraine, Ukraine.
} 


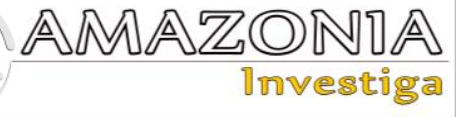

Key words: science, terminology, metaphor, representation, popular science.

термінів у різних дисциплінах 3 метою кращого розмуміння ї впливу на розвиток наукового знання як експертами, так i неспеціалістами.

Ключові слова: наука, термінологія, метафора, репрезентація, науковопопулярний дискурс.

\section{Introduction}

Over the last four decades, the pivotal significance of metaphor in human communication and reasoning has come into the limelight. It is recognized today not merely as a rhetorical flourish but as a fundamental conceptualization tool helping us to interpret and categorize the manifold phenomena and objects around us. In their seminal book "Metaphors We Live By" George Lakoff and Mark Johnson argue, "Metaphor is pervasive in everyday life, not just in language but in thought and action" (1980, p. 3). We especially rely on metaphor when having to deal with abstract concepts that are hard to explain or understand otherwise. According to the Conceptual Metaphor Theory, abstract concept is typically represented as more concrete concept via cross-domain mapping from the source domain to the target domain (Lakoff \& Johnson, 1980; Lakoff \& Johnson, 1999).

Contrary to the widespread opinion, scientific discourse is not devoid of metaphor. Given that scentists have to operate highly abstract notions, which are hard to illustrate and impossible to grasp even for experts (particularly as far as modern quantum mechanics is concerned), they are often left with no other option than to resort to metaphor, albeit not always willingly. As Jon Turney (2001) puts it, "Science wants to be precise, unambiguous, logical and universal. Natural language is none of these things: hence scientists' addiction to mathematics. But if they cannot manage with words alone, neither can they do without them. Words lend coherence, build understandable narratives, explain what the mathematics is about". Prominent scientists, including Albert Einstein and Charles Darwin, believed that metaphor is crucial to the development of new scientific ideas, and the history of science over the centuries bears out this claim (Montuschi, 2017, p. 277). The fact that metaphors tend to serve as a basis for the invention of scientific theories has been underlined by many researchers including Max Black (1993), Richard Boyd (1993), Alan Lightman (1989), Richard Johnson-Sheenan (1997), Thomas Kuhn (1993), Stuart Peterfreund (1994) and others. Moreover, apart from its

heuristic value, metaphor in science also has a crucial pragmatic function as it guides the scientific inquiry and suggests directions in which to look (Haack, 2019, p. 2060). However, metaphors in science also pose some problems as they can distort the true knowledge and lead to public misundrstandings (Taylor \& Dewsbury, 2018). The purpose of this article is to take a closer look at some of the key metaphor-induced terms in modern astrophysics and cosmology as a fast-developing area of scientic inquiry that has many repercussions in popular culture. We intend to analyze the mapping structure and the pragmatic value of these metaphors, while also exploring their limitations, which sometimes lead to inaccuracies and misinterpretations. In this way we hope to contribute to understanding of the linguistic factors involved in the development of science as an enterprise having far-reaching implications for everyone.

\section{Literature review}

Metaphor and analogy in scientific discourse have predominantly been explored in the context of science education and communication. The theory of conceptual metaphor, in particular, has been applied to a wide range of physical concepts, such as entropy (Amin et al., 2010, Haglund, Jeppson, \& Strömdahl, 2010), energy (Lancor, 2014), elementary particles (Ceroni, 2014), heat and motion (Corni, Fuchs \& Dumont, 2019), various concepts of quantum mechanics (Brookes \& Etkina, 2007). Jennifer Burwell draws on the Conceptual Metaphor Theory to analyze the concepts of quantum mechanics and trace their transformations through time in her recent book "Quantum Language and the Migration of Scientific Concepts" (2018). In another relevant study, Richard JohnsonSheenan (1997) analyzes Planck's 1900 Quantum Paper showing how it introduced a powerful new metaphor, 'energy is quantized', which revolutionized science. The researcher emphasizes the fact that metaphorical analysis can have special value in exploring the rhetoric of revolutionary texts in science. With regard to cosmology, analogical and metaphorical 
reasoning in this field has been investigated by Gustaaf Cornelis (2000), who demonstrates significant reliance of cosmological thought on analogies in devising new theories, based on the analysis of the notions of black hole, event horizon and stellar evolution.

Another strand of research has concerned itself with the significance of metaphor in terminology construction. Metaphoric strategies in the formation of scientific vocabulary first came under linguistic scrutiny in the 1980s when the common derivation from Greek and Latin roots no longer defined scientific terminology (Raad, 1989). In one of the first papers devoted to metaphors in physics in particular - "Physics as Metaphor and Vice Versa" - Frieda Stahl aptly notes: "The metaphoric process is as pervasive in Western physics as in Western literature." (1987, p. 58). She points out that phycisists often find a metaphoric term, which, despite some initial insight, has denotative limitations and thus the metaphor is soon rendered useless. She refers to "particle" and "wave" as the most salient examples of this phenomenon: both provide inadequate description of the respective physical entities but were called this way due to the lack of a better option. (ibid., 58-59). Numerous cases when metaphorical models in physics show discrepancy with the actual referents have also been profoundly analyzed in the book "Aspects of Metaphor in Physics: Examples and Case Studies" by Hanna Pulaczewska (2011). Thus, she finds faults with the concept of "state" and with the modelling of probabilities as spreading waves as examples of entity-making metaphors, which accommodate our thing-based image of the world and provide a sense of cognitive satisfaction while remaining very superficial. The book draws heavily upon the important distinction between theory-constitutive and pedagogical (educational) metaphors made by Richard Boyd (1993). Theory-constitutive metaphors take an irreplaceable part in the theory development as they offer no other path of understanding the concept besides the metaphorical conceptualization ("electricity is a fluid"), while pedagogical metaphors merely facilitate the teaching of physical concepts and may show great variability ("atoms as miniature solar systems"). All the units analyzed in our article represent theory-constitutive metaphors as they have become terminologically solidified (not just occasionally used) and have influenced further development of scientific theories and approaches. As argued by Hanna Pulaczewska, "theory-constitutive metaphors of modern physics pose as many questions as they appear to answer" (2011, p. 201). In our article, we intend to contribute to the mounting literature on the importance and ambiguity of such metaphors in scientific discourse.

\section{Methodology}

For the purposes of this article the qualitative approach was used. We browsed the headlines of cosmology and physics articles on credible scientific news portals (particularly, Science Daily, Scientific American, Science News and Quanta Magazine) over the last two years and compiled a list of frequently featured scientific terms based on cross-domain metaphorical mapping. Most of them consisted of a single lexeme, while others were represented with two lexemes (namely, "big bang", "black hole"). Each term was searched in Google Scholar to verify its broad and established use in academic sources in respective areas. We traced the origin of these terms, analyzed their etymology and conceptual structure, and (for some units) detected incongruencies in their nominalizations and essential features of the referenced object (the so-called misnomers). We also paid attention to the reinterpretations of these units in academic discourse and popular culture and evaluated the most notable criticisms leveraged against them at present, drawing upon specialized literature in the field.

\section{Results and Discussion}

\section{"Big Bang”}

It would seem reasonable to begin with the very beginning of the universe - the "Big Bang" which is one of those few scientific terms well known to any average person, no matter how far from science, due to its frequent depictions in popular culture. In fact, this term, which paints a vibrant picture of the universe being born in a fantastic explosion, was coined humorously by English cosmologist Fred Hoyle in a 1949 BBC interview to refer to a theory that he was ardently opposed to (Mitton, 2011; Kragh, 2013). He advocated the steady-state model, whereby the density of matter in the universe, and therefore its appearance, remains the same over time due to the constant creation of new matter. The competing theory of "primeval atom", developed by $\mathrm{G}$. Lemaître, maintained that the universe expanded from one initial point (which is now called "singularity"). The empirical evidence gradually lended almost irrefutable support to the latter theory, which came to be known as the "Big Bang" / "big bang" both in reviewed physics journals and science popularizations. 


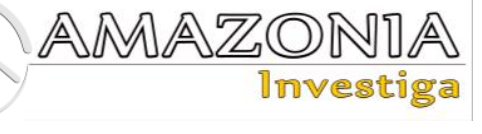

As it remains the predominant cosmological model now, the term is used ever more often. A search in Google Scholar yields 1020000 results, which is quite impressive. And that's despite the fact the term is universally agreed today to be a misnomer (Kragh, 2013). As explained by Luke Mastin, "the Big Bang was neither Big (in the beginning the universe was incomparably smaller than the size of a single proton), nor a Bang (it was more of a snap or a sudden inflation)" (Mastin, 2019, p. 25). He goes on to claim that the analogy of explosion is not relevant here at all as it implies that the birth of the universe started at some particular center, while in reality the identical pattern of expansion could be observed from any point (ibid., 25-26). We can also add that an explosion usually leads to destruction, whereas this cosmic event was constructive, bringing about the creation of matter, space and time. Even though the term obviously lacks precision, it has not only taken over the physics world, but also has given birth to several other metaphorical terms following the same syntactical model. When addressing the ultimate fate of the universe, scientists envision quite differing potential scenarios, namely Big Crunch (the Universe will shrink and collapse in on itself in reversal of the Big Bang), Big Rip (the Universe will be torn apart), Big Freeze/Big Chill (the Universe will become unable to sustain motion reaching a "heat death") (Mack, 2020). One can also come across other less common terms like "Big Slurp", "Big Snap", "Big Bounce" (they keep proliferating as the topic is drawing increasing attention in astrophysics). Thus, "big bang" greatly illustrates how an occasionally (and even humorously) coined metaphorical term in science can become firmly established to the extent of shaping further terminological development in the area.

\section{"Wormhole"}

Another important metaphor-induced term in astophysics and cosmology is "wormhole", which is well known today even to those outside of this field due to the raging popularity of the 2014 science fiction film "Interstellar". Wormhole is a theoretically envisaged passage though spacetime that creates shortcuts in the universe. While wormholes have not been observed in reality so far, their existence is possible from the mathematical viewpoint. The notion is also known in physics as "EinsteinRosen bridge", after the physicists Albert Einstein and Nathan Rosen who predicted this phenomenon based on the general theory of relativity. However, this bland eponymic term is rarely to be found nowadays, being universally replaced with "wormhole", which was coined by American physicist John Archibald Wheeler in 1957 (in an article co-authored with Charles Misner). He presented it in the following way: "This analysis forces one to consider situations [...] where there is a net flux of lines of force, through what topologists would call 'a handle' of the multiply-connected space, and what physicists might perhaps be excused for more vividly terming a "wormhole" (Misner \& Wheeler, 1957, p. 532). The vehicle of the metaphor in this case is the narrow passage burrowed by an insect or worm in fruit or wood. It enables a creature to turn up on the other side of an object much faster than by moving on the surface, just like the quantum wormhole potentially grants the opportunity to reach a destination that is light-years away in the general structure of spacetime. In his explication of this notion for Scientific American, William A. Hiscock constructs an extended metaphor urging the readers to imagine an ant crawling through a wormhole in an apple. Moreover, he points out that while cosmic wormholes are usually assumed to be shortcuts, they may actually represent a longer route similarly to complex and twisting burrows that worms sometimes make (Scientific American, 1997). Thus, the metaphor turns out to be accurate enough to capture even such subtle commonalities as the variations in length of the passages. Apart from its accuracy, however, it also carries mysterious (a wormhole is deeply hidden) and escapist (a worm can run away through it) overtones that have spurred abundant speculations of time travel and paranormal phenomena, furnishing rich material for science fiction literature and cinema. Would the film "Interstellar" be possible if "wormholes" (discussed there at much length) were known exclusively as "Einstein-Rosen bridges"? We doubt it.

\section{"Black hole"}

Another term that is dominating modern physics and permeating popular science is "black hole", defined as a region of spacetime with an extremely strong gravitational pull, which does not let any particles or light escape from it. The size of black holes varies heavily, some being giant, others the size of an atom. Since the beginning of the 20th century, when this phenomenon was first predicted, they went under different names such as "dark star", "gravitationally collapsed object", "completely collapsed gravitational object". The term "black hole" was coined by the physicist Robert Dicke circa 1960 when he drew the analogy of this peculiar object to the Black Hole of Calcutta, a 
small prison notorious for its cramped conditions, where on one occasion almost all prisoners were suffocated (Herdeiro \& Lemos, 2018 , p. 9-10). However, the term only started to be taken seriously after it had been granted the authority of the above-mentioned physicist John Wheeler. At a conference in New York in 1967 he had to mention the term "gravitationally completely collapsed object" a few times and commented on its bulkiness, expressing the need for a shorter alternative. A person from the audience (some sources suggest it was Robert Dicke himself) shouted "black hole" and Wheeler gladly picked it up, going on to use it in his subsequent articles (Herdeiro \& Lemos, pp. 6-7). Despite some resistance the term met at the beginning of its use (notably from the renowned phycist Richard Feynman), it was soon widely accepted in the academia. At present, there is no alternative to it. The problem, however, is that a black hole is not actually a hole at all: it is a region just like any other apart from its extreme gravity. Moreover, it cannot be precisely described as "black": it is just invisible because light cannot escape from it. So neither the attribution of color nor the imposed imageschema of containment seems to be in accordance with the inherent characteristics of this space object.

The nominalization of "black hole", which affords many conjectures from overly imaginative minds, is to a large extent responsible for the common personification of black holes and depiction of them as monsters in popular culture. This has become the focus of Carolyn DeCristofano's book for young readers "A Black Hole Is Not a Hole" (2017). In the introduction, the author aptly observes: "From the headlines, you'd think black holes were beasts with endless appetites, lying in wait for the next meal. By some reports they are "runaway," out-of-control "predators" that "feed" on galaxies, only to "belch" and "spit out" what they don't eat. They "lurk" in the shadows, "mangling" stars and "gobbling" them up. In short, they have a nasty reputation for being monsters "gone mad." (DeCristofano, 2017, p.1). Hence, the more imaginative the term, the more food it provides for popular culture, which supplies further details to the image, often distorting it.

\section{"Spaghettification"}

The common representation of black holes as hungry mosters has found its reflection in another metaphor-induced scientific term "spaghettification" (sometimes also referred to as "the noodle effect"). The term denotes the process of stretching and compression of an object due to extreme tidal forces, with its inevitable destruction. The object thus becomes shredded into thin long stretches that resemble spaghetti. It was first used in the late 1970s but only gained momentum after Stephen Hawking mentioned it in his bestseller book "A Brief History of Time" in the vivid and painfully detailed account of a fictional astronaut falling into a black hole: "Gravity gets weaker the farther you are from the star, so the gravitational force on our intrepid astronaut's feet would always be greater than the force on his head. This difference in the forces would stretch our astronaut out like spaghetti or tear him apart before the star had contracted to the critical radius at which the event horizon formed!" (Hawking, 1988, p. 88).

It was exactly Hawking who sparked an intense interest in the topic of black holes among scientists and laypeople alike. The vast popularity of his book reinforced the position of "spaghettification" as a staple of astrophysics jargon. Moreover, it has become known to educated audience since 2019 when the actual colossal event of a star being spaghettified during a tidal disruption (known as AT2019qiz) was filmed for the first time, testifying to the accuracy of the scientists' predictions. Notably, some mass media accounts of this event played with the cuisine metaphor inherent in the term "spaghettification" extending it further with impressive personifications of the "hungry" black hole, e.g.: "A black hole enjoyed one stellar spaghetti dinner and astronomers were able to witness the event from 215 million light-years away in a spiral galaxy in the Eridanus constellation" (Strickland, 2020) and the headline "A Black Hole's Lunch: Stellar Spaghetti. Astronomers observed a star become a "feast" for a cosmic monster." (Overbye, 2020). So in this case, too, the term's metaphoricity serves to fuel creativity in layman's popularizations of scientific notions and reach wider audience.

\section{"Gravitational hair"}

Having a great appetite is not the only humanlike trait that is indirectly attributed to black holes: in cutting-edge physics studies, they are also revealed to possess "hair" (featured in the metaphorical terms "gravitational hair", "soft hair" and "quantum hair"). The history of these terms can be traced back to John Wheeler (again), who once said the phrase "black holes have no hair", implying that all black holes are 


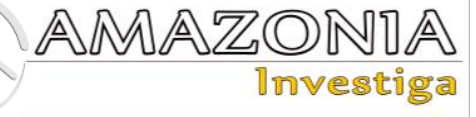

extremely simple and can be fully characterized by three parameters: angular momentum, mass, and electric charge. All the other information disappeared beyond the event horizon (Ghose, 2016). Thus, "hair" was meant as a metaphor for complicated and unique features that a black hole lacks similarly to a hairless head lacking distinctive traits. This view came to be known as "no-hair theorem" or "no-hair conjecture", which is a curious illustration of how occasional imaginative remarks become solidified in scientific terminology.

Moreover, this metaphor was picked up even further and given new life in recent scientific developments. Thus, one of the last studies authored by Stephen Hawking (with colleagues) introduced the concept of "soft hair", which points to the additional properties that black holes might have (Hawking, Perry \& Strominger, 2016). The word "soft" here is related to the photon wih vanishing energy (soft photons) that basically enables this effect. In later studies the terms "quantum hair" and "gravitational hair" also came into use (see, for example, Lior et al., 2021, Brustein \& Medved, 2018). Along the way, these terms have also given birth to numerous derivative expressions such as "hairy black holes", "hairless law", "soft-haired model". Furthermore, the metaphor is often extended with extra features in science popularizations, e.g. "Extreme black holes have hair that can be combed" (Theiss Research, 2021), "Nearly extreme black holes which attempt to regrow hair become bald again" (Theiss Research, 2019).

\section{"Fuzzball”}

In string theory, black holes are reimagined in a peculiar way and dubbed with yet another metaphorical coinage, "fuzzball". Fuzzballs are posited as entangled balls of strings (vibrating bundles of energy) that have no singularity and no event horizon (which are key attributes of black holes in classical theory) (Ouelette, 2015). In place of event horizons, fuzzballs have "fuzzy" surface, hence their name, invented by Samir D. Mathur (2005). This representation is far less scary than that of a "black hole" and invokes the image of a fluffy toy (known under the same name, also "a puffball"). They are not empty holes but dense objects with a surface resembling that of a star or a planet. They emit radiation, which means that information is not lost in them irretrievably (which is postulated for classical black holes). Here the "benign" nominalization turns out to be reflective of the more profound benign nature of the referred entity. "Fuzzbalss" are not totally harmless but they are definitely not the hungry monsters that the name "black hole" invites one to imagine.

\section{"String”}

Speaking about the string theory, it is worth explicating its central notion in more detail as in this case the underlying metaphor seems to affect the theory construction to a large extent. String can be defined as a vibrating one-dimensional entity having various vibrational modes, each corresponding to a different particle. The common image used to explicate this concept to a layperson is a violin string (see, for example, vivid introduction by one of the leading advocates Brian Greene, 2019). However, this analogy is far from being perfect. Jon Turney (2001) acutely observes: "No sooner are we dusting off our intuitions about violin strings than the image undergoes violent alteration. For these strings can never be bowed or plucked. They are almost inconceivably small, 1020 times smaller than an atomic nucleus. [...] Nor are these strings stretched between fixed points like those in a musical instrument because, again like rubber bands, they are looped around." Moreover, for now, it is believed that quantum strings are not made of anything, they are the building blocks of matter in themselves. However, according to Turney, by far the most salient feature that does not sit well with the word "string" at all, is the ability of individual strings to merge or divide. Therefore, essentially, the only quality that serves as the common ground for the vehicle and the tenor of this metaphor is vibration (or oscillation), which produces different results (notes or particles) in different modes. In all other respects, a physical string is too distant an analogy for the massless objects postulated by the string theory. Despite its conceptual inadequacy, this metaphorical conceptualization has gone a long way in promoting specialist knowledge in the field to the lay public. Clinging onto the commonly used violin string analogy and building upon it, popular accounts of string theory tend to employ numerous musical metaphors involving the celestial harmony, music of the spheres, cosmic symphony and the like. Alex Volmar and Peter Pesic (2014) explain that string theorists appeal to music as universal affective experience in trying to "sell" their theory (which is much disputed and has an army of opponents) to the lay public. This way, they are trying to persuade the audience (upon whom their funding is dependent to some extent) that the string theory is exactly the "theory of everything" (theory unifying the description of gravity and particle physics) that researchers 
have long searched for. Hence, the metaphor underlying a scientific term can become the centrepiece of rhetoric strategy employed by scientists, however imprecise it may be.

\section{Conclusions}

Having analyzed the most common metaphorinduced terms in modern astrophysics and cosmology with a close attention to the correlation between their source and target domains, we can see that scientific metaphors in this field are often inaccurate or limited and thereby they may produce or reinforce misconceptions about the referred notions. The overwhelmingly popular terms "big bang", "black hole" and "string" (in string theory) seem to be the most problematic (and it has been already recognized by physicists). The event described by big bang was not big, neither was it abrupt or destructive akin to an explosion. Nevertheless, the term is so firmly established that it has given rise to many units constructed in the same pattern ("big chill", "big freeze", etc.). The black hole imposes the image-shema of containment upon a space region with extreme gravity that basically cannot contain anything. String in string theory is very unlike a string of a musical instrument in that it can merge or divide. Furthermore, it is not made of anything, while a typical string is made of some material.

We have also revealed that inherent metaphoricity of some scientific terms is often extended further in layman's accounts, in accordance with Jon Turney's observation that key metaphors from professional discourse "are invariably enriched and elaborated in new ways as popular accounts of new theories proliferate" (2001). Thus, reports on spaghettification events utilize further food metaphors and personify black holes as hungry monsters, while popular books on string theory are peppered with extensive musical metaphors. While these extended metaphors may be severely lacking in accuracy, they serve an important pragmatic function of engaging the audience with science. Still, caution is needed as they can be highly misleading, imposing wrong representations upon the lay public, as well as the researchers, who involuntarily succumb to the mental picture invoked by provided metaphors. Further research in this area could focus on the psycholinguistic aspects of metaphor processing in science. It would also be worthwhile to analyze metaphorical terms from other disciplines such as chemistry, biology and medicine.

\section{Bibliographic references}

Amin, T., Jeppson, F., Haglund, J., \& Strömdahl, H. (2010). Metaphorical resources for understanding entropy and the second law of thermodynamics. Paper presented at the 7th Biennal Meeting of the Conceptual Change Special Interest Grouip, European Association for Research on Learning and Instruction, Leuven, Belgium, May 24-27th, 2010.

Black, M. (1993). More about metaphor. In Ortony, A. (Ed.), Metaphor and thought (pp. 19-41). Cambridge: Cambridge University Press.

Boyd, R. (1993). Metaphor and theory change: what is metaphor a metaphor for? In Ortony, A. (Ed.), Metaphor and thought (pp. 356408). Cambridge: Cambridge University Press. doi: 10.1017/CBO9781139173865.023

Brookes, D.T., \& Etkina, E. (2007). Using conceptual metaphor and functional grammar to explore how language used in physics affects student learning. Physical Review Special Topics - Physics Education Research, 3(1), 1-16.

Brustein, R., \& \& Medved, A. J. M. (2018). Quantum hair of black holes out of equilibrium. Physical Review. D., 97(4), doi: 10.1103/physrevd.97.044035

Burwell, J. (2018). Quantum language and the migration of scientific concepts. Cambridge, MA: MIT Press.

Ceroni, G. (2014). Exploring the Use of Metaphor in Communication of Contemporary Physics. Procedia - Social and Behavioral Sciences, 116, 1324-1332. doi: 10.1016/j.sbspro.2014.01.391

Cornelis, G. (2000). Analogical Reasoning in Modern Cosmological Thinking. In F. Hallyn (Ed.), Metaphor and analogy in the sciences (pp. 165-180). Springer.

Corni, F., Fuchs, H. \& Dumont, E. (2019). Conceptual metaphor in physics education: roots of analogy, visual metaphors, and a primary physics course for student teachers. Journal of Physics: Conference Series, 1286, 012059 doi: 10.1088/17426596/1286/1/012059.

DeCristofano, C. (2017). A black hole is not a hole. Watertown, MA: Charlesbridge Publishing.

Ghose, T. (2016). Stephen Hawking: Black Holes Have 'Hair'. Live Science. Retrieved from https://www.livescience.com/53363stephen-hawking-black-holes-have-hair.html 


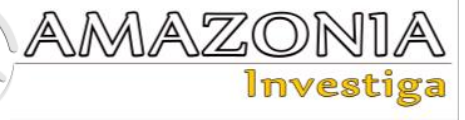

Greene, B. (2019). What is String Theory? World Science Festival. Youtube. Retrieved from https://www.youtube.com/watch?v=TI6sY0 $\mathrm{kCPpk}$

Haack, S. (2019). The art of scientific metaphors. Revista Portuguesa de Filosofia, 75(4), 20492066. doi: 10.17990/RPF/2019_75_4_2049

Haglund, J., Jeppson, F., \& Strömdahl, H. (2010). Different senses of entropy Implications for education. Entropy, 12(3), 490-515.

Hawking, S. (1988). A Brief History of Time. Bantam Dell Publishing Group.

Hawking, S., Perry, M. J., \& Strominger, A. (2016). Soft Hair on Black Holes. Physical Review Letters, 116(23), 231301.

Herdeiro, C.A., \& Lemos, J.P.S. (2018). The black hole fifty years after: Genesis of the name. Gazeta de Fisica, 41(2), 2. Retrieved from https://arxiv.org/abs/1811.06587

Johnson-Sheehan, R. D. (1997). The Emergence of a Root Metaphor in Modern Physics: Max Planck's "Quantum" Metaphor. Journal of Technical Writing and Communication, 27(2), 177-190. doi: 10.2190/lxwh-uxtl2bbt-prm5

Kragh, H. (2013). Big Bang: the etymology of a name. Astronomy \& Geophysics, 54(2), 2.28-2.30. doi: 10.1093/astrogeo/att035

Kuhn, T. (1993). Metaphor in science. In Ortony, A. (Ed.), Metaphor and thought (pp. 533542). Cambridge: Cambridge University Press.

Lakoff, G., \& Johnson, M. (1980). Metaphors we live by. Chicago: University of Chicago Press.

Lakoff, G., \& Johnson, M. (1999). Philosophy in the flesh: The embodied mind and its challenge to western thought. New York: Basic Books.

Lancor, R. (2014). Using metaphor theory to examine conceptions of energy in biology, chemistry, and physics. Sci \& Educ, 23, 1245-1267. doi: 10.1007/s11191-012-95358

Lightman, A. (1989). SCIENCE: Magic on the mind: physicists' use of metaphor. The American Scholar, 58(1), 97-101.

Lior, M. B. et al. (2021). Scalar and gravitational hair for extreme Kerr black holes. Phys. Rev. D $\quad 103 \quad$ (2), L021502. doi: 10.1103/PhysRevD.103.L021502

Mack, K. (2020). The End of Everything: (Astrophysically Speaking). Scribner.

Mastin, L. (2019). The Big Bang and the Big Crunch. In Noble, F.R., Cooper, T.M., \& Mullat, J.E. (Eds.), Cosmology that contradicts the big bang theory (pp. 25-77).
Hvidovre, Denmark: Private Publishing Platform.

Mitton, S. (2011). Fred Hoyle: A life in science. Cambridge University Press.

Mathur, S. D. (2005). The fuzzball proposal for black holes: An elementary review. Advances in physics, 53, 793 - 827. doi: 10.1002/prop.200410203.

Misner, C. W., \& Wheeler, J. A. (1957). Classical physics as geometry. Ann. Phys., 2(6), 525-603. doi: 10.1016/00034916(57)90049-0.

Montuschi, E. (2017). Metaphor in science. In Newton-Smith, W.H. (Ed.). A Companion to the Philosophy of Science. Blackwell Publishers. Doi: https://doi.org/10.1002/9781405164481.ch4 1

Ouelette, J. (2015, June 23). The Fuzzball Fix for a Black Hole Paradox. QuantaMagazine. Retrieved from https://www.quantamagazine.org/howfuzzballs-solve-the-black-hole-firewallparadox-20150623/

Overbye, D. (2020, Oct. 17). A Black Hole's Lunch: Stellar Spaghetti. The New York Times. Retrieved from https://www.nytimes.com/2020/10/17/scienc e/astronomy-black-hole-at1910qix.html

Peterfreund, S. (1994). Colonization by means of analogy, metaphor, and allusion in Darwinian discourse. Configurations, 2(2), 237-255. Doi: $10.1353 /$ con.1994.0029.

Pulaczewska, H. (2011). Aspects of metaphor in physics: Examples and case studies. Berlin, New York: Max Niemeyer Verlag.

Raad, B. (1989). Modern trends in scientific terminology: Morphology and metaphor. American Speech, 64(2), 128-136. doi: 10.2307/455039

Scientific American (1997). What exactly is a 'wormhole'? Have wormholes been proven to exist or are they still theoretical? Scientific American. Retrieved from https://www.scientificamerican.com/article/f ollow-up-what-exactly-is/

Stahl, F. A. (1987). Physics as metaphor and vice versa. Leonardo, 20(1), pp. 57-64.

Strickland, A. (2020, Oct.12). Astronomers witness 'spaghettification' of star shredded by a black hole. CNN. Retrieved from https://edition.cnn.com/2020/10/12/world/sta r-spaghettification-black-hole-death-scntrnd/index.html

Taylor, C., \& Dewsbury, B. M. (2018). On the problem and promise of metaphor use in science and science communication. Journal of microbiology \& biology education, 19(1), 19.1.46. doi: 10.1128/jmbe.v19i1.1538 
Theiss Research (2019). Nearly extreme black holes which attempt to regrow hair become bald again. PhysOrg. Retrieved from https://phys.org/news/2019-11-extremeblack-holes-regrow-hair.html

Theiss Research (2021). Extreme black holes have hair that can be combed. PhysOrg. Retrieved from https://phys.org/news/202101-extreme-black-holes-hair.html
Turney, J. (2001). Strings and things. Nature, 410, 873. doi: $10.1038 / 35073712$

Volmar, A. \& Pesic, P. (2014). Pythagorean Longings and Cosmic Symphonies: The Musical Rhetoric of String Theory and the Sonification of Particle Physics. Journal of Sonic Studies, 8. Retrieved from www.researchcatalogue.net/view/109371/10 $9372 / 0 / 53$ 